\title{
Effect of anharmonicity on energy relaxation of a dissipative quantum oscillator
}

\author{
RAMESH KUMAR and PRADEEP KUMAR*(i) \\ Department of Chemistry, Malaviya National Institute of Technology Jaipur, Jaipur 302017, India \\ E-mail: pradeep.chy@mnit.ac.in
}

MS received 23 July 2018; revised 25 January 2019; accepted 31 January 2019; published online 2 March 2019

\begin{abstract}
The effect of anharmonicity on the energy relaxation of excited state has been studied by means of a dissipative oscillator. The present study also sheds light on the recent femtosecond stimulated Raman spectroscopy results of Kovalenko et al. (J. Chem. Phys. 139011101 2013) on optical cooling of trans-stilbene. It has been shown that anharmonicity plays a crucial role in explaining the time-dependent frequency shift as well as the time evolution of bandwidth of $v_{C=C}$ mode of trans-stilbene in excited state, found in experiment.
\end{abstract}

Keywords. Stilbene; anharmonicity; relaxation.

\section{Introduction}

It is known that if one excites a molecule in the presence of a bath (everything except system), the molecule will eventually come to a state of equilibrium by exchanging energy with bath. This energy relaxation is key to several phenomena that are important in physics as well as in biology. In physics, it becomes important in the study of problems like, vibrational energy relaxation of large molecules in liquids, ${ }^{1}$ energy relaxation in quantum dots, ${ }^{2}$ surface relaxation in organic semiconductors, ${ }^{3}$ etc. In biology, it is key to the understanding of very basic phenomena like photosynthesis and animal vision. In photosynthesis, plant uses light to photosynthesize food, which reaches us in different forms. Photosynthetic conversion of solar energy into chemical energy, suitable for cellular processes, involves a variety of physiochemical reactions. At low light intensities, the quantum efficiency of this process is found to be very high. ${ }^{4}$ A longstanding question in physics is, why is the efficiency of photosynthetic conversion so high in the presence of such a complicated environment? In case of vision, the key step is, photochemical cis-trans isomerization of 11-cis retinal chromophore in rhodopsin. ${ }^{5}$ Quantum yield of this reaction is almost $0.65 .^{6}$ One can again ask, why in such complicated environment, quantum yield is so high? To shed light on these questions,

\footnotetext{
*For correspondence
}

study of energy relaxation phenomenon becomes very important.

There are two major bottlenecks in the theoretical study of biological reactions in condensed phase: First, biological molecules are often very large in size, therefore, their direct study is computationally very demanding. Second part of the problem lies in accurate modeling of the environment. In modeling of the environment, harmonic bath model have gained a lot of popularity in deriving numerical as well as analytical results. ${ }^{7-10}$ To circumvent the first bottleneck, one uses some prototype molecules. Similar to vision process, a lot of biologically important processes involve cis-trans isomerization. In this regard, trans-stilbene is considered as one of the important prototype molecule to study cis-trans photoisomerization processes. ${ }^{11-13}$

Particularly, the study of photoisomerization of stilbene in solution was found to be very useful in understanding the role of solvent in chemical reactions in general. The isomerization rate of trans-stilbene in low pressure gas was found to vary from $4.7 \times 10^{8} \mathrm{~s}^{-1} \mathrm{bar}^{-1}$ (in helium) to $7.1 \times 10^{9} \mathrm{~s}^{-1} \mathrm{bar}^{-1}$ (in propane). ${ }^{14}$ The same in solution becomes greater than $10^{11} \mathrm{~s}^{-1} \cdot \cdot^{15,16}$ The question, why is isomerization rate in solution phase much higher than gas phase, was a matter of number of theoretical and experimental studies. ${ }^{14,17-19}$ Different answers were suggested by various research groups periodically. Based on the fluorescence lifetime measurements, Fleming and co-workers, conjectured that reaction becomes adiabatic in the solution phase, which accounts for a higher rate in solution. ${ }^{16,17,20}$ 
Troe et al., suggested that, barrier height for isomerization of trans-stilbene might become lower in solution phase due to clustering. ${ }^{14,21} \mathrm{~A}$ fast intramolecular vibrational redistribution (IVR) in solution as compared to gas phase due to solute-solvent interaction was also proposed as a cause of this effect. ${ }^{17,22}$ In a series of papers, Pollak and co-workers tried to explain it, based on the concept of nascent cooling of excited stilbene molecule. ${ }^{18,19}$ They argued that, the effective vibrational temperature of an excited molecule is less than the actual temperature, therefore, the rate is slow in the gas phase. Whereas, in solution phase molecules get reheated by solvent molecules which increases the rate in solution phase. ${ }^{18,19,23,24}$ Besides these, earlier Hamaguchi and co-workers ${ }^{25}$ and recently Kovalenko et al. ${ }^{26}$ used time-dependent Raman spectroscopy to study the relaxation of trans-stilbene in solution phase.

The Hamaguchi group used time-resolved Raman spectroscopy to study the isomerization of trans-stilbene in $S_{1}$ state in solution phase. ${ }^{27-29}$ They found a linear correlation between bandwidth and peak position in the Raman spectra of the $v_{C=C}$ (stretching frequency of $-C=C-$ ) stretching mode of transstilbene. They also measured the bandwidth and peak position using various solvents ( hexane, octane and decane) ${ }^{28}$ Their spectroscopic data indicate that, this linear correlation between bandwidth and peak position persists in all three solvents. They proposed that, if this energy relaxation in trans-stilbene experiment can be understood properly, then picosecond timeresolved fluorescence spectroscopy can be used as a picosecond Raman thermometry. To explain, the linear correlation between peak position and bandwidth, Hamaguchi and co-workers proposed a "dynamical polarization model (DPM)". ${ }^{25,28}$ In this model, it was assumed that, the width of the peak is the consequence of frequency modulation of $v_{C=C}$ mode due to solvent.

Recently Kovalenko et al., studied the optical cooling of trans-stilbene in $n$-hexane solvent in femtosecond domain, ${ }^{26}$ using femtosecond stimulated Raman spectroscopy technique (FSRS) at various initial energies. ${ }^{6}$ Interestingly, they found that the relaxation path depends strongly on the initial energy. When, they choose the initial energy such that the excited molecule remains in vibrational ground state, they found a linear correlation between bandwidth and peak position which was similar to earlier results. But, when they decreased the initial wavelength (excited molecule is in vibrationally excited state) the bandwidth first increases for few picoseconds and then decreases monotonically to the asymptotic equilibrium state. In the rest of the manuscript, this work is abbreviated as FSRS experiment.
DPM explains the experimental results quite well in picosecond domain but fails in femtosecond region. It is not surprising, as DPM is a phenomenological model, it was designed, to capture the experimental fact in picosecond domain. ${ }^{25,28}$ Therefore, when the same experiment was carried out in femtosecond domain, and a non-linear correlation between bandwidth and peak position was found, the model fails.

The experimental facts mentioned in the preceding paragraph are very generic. An understanding of these experimental facts from first principle will be very useful in understanding the role of solvent in condensed phase chemical reactions.

There are few theoretical studies, regarding energy relaxation of trans-stilbene molecule. ${ }^{18,19,30,31}$ But to the best of our knowledge only Kumar et al., attempted to study the effect of initial energy on the relaxation bath of $v_{C=C}$ mode of trans-stilbene in $S_{1}$ state. Kumar et al., used the Hamiltonian based Langevin model to study the energy relaxation of harmonic oscillator. ${ }^{7}$ They derived the analytical expression for time-dependent average energy as well as energy fluctuations in continuum limit. They also derived the analytical expression for the population spectrum. They indicated in their paper that, their model might be useful in understanding the energy relaxation phenomena in general. A limitation of their model was, they assumed the system to be harmonic. Tatchen et al. ${ }^{24}$ indicates that theoretical understanding of isomerization of trans-stilbene will be incomplete without the incorporation of the effect of anharmonicity. In the present work, we extended the result by Kumar $e t$ $a l .,{ }^{7}$ by including anharmonicity and further used it to shed light on the results of FSRS experiment.

\section{Theory}

Kumar et al., used the following Hamiltonian to describe the energy relaxation process in excited state. ${ }^{7}$

$\hat{H}=\frac{1}{2}\left(\hat{p}_{q}^{2}+\Omega_{1}^{2} \hat{q}^{2}+\sum_{j=1}^{N}\left[\hat{p}_{j}^{2}+\omega_{j}^{2}\left(\hat{x}_{j}-\frac{c_{j}}{\omega_{j}^{2}} \hat{q}\right)^{2}\right]\right)$

Where, $\hat{p}, \hat{x}, \hat{p_{q}}$, and $\hat{q}$ are mass weighted momentum and position operators of bath and system respectively. The system was assumed to be a harmonic oscillator with frequency $\Omega_{1}$ and bath was a bunch of harmonic oscillators $\left(\omega_{1}, \omega_{2} \ldots \omega_{n}\right)$. The coupling between system and bath was assumed to be linear. The bath was characterized by a spectral density $J(\omega)$, which can be obtained from a time dependent friction function $\gamma(t)$, i.e. 
$J(\omega)=\omega \int_{0}^{\infty} d t \cos (\omega t) \gamma(t)$

It was assumed that at the beginning of the process the system was not coupled to the bath i.e., the initial state was factorized. Thus the system's initial state was taken to be a Gaussian and bath was assumed to be thermally distributed. ${ }^{7}$

Consequently, their initial density $\left(\rho_{0}\right)$ was of the following form,

$\hat{\rho}_{0}=|\Psi\rangle\langle\Psi| \frac{\exp \left(-\beta \hat{H}_{B}\right)}{\operatorname{Tr}\left[\exp \left(-\beta \hat{H}_{B}\right)\right]}$,

where, $|\Psi\rangle$ was defined as

$$
\begin{aligned}
& \left\langle q \mid \Psi\left(q_{0}, p_{0}\right)\right\rangle \\
& =\left(\frac{\Omega_{0}}{\pi \hbar}\right)^{1 / 4} \exp \left(-\frac{\Omega_{0}\left(q-q_{0}\right)^{2}}{2 \hbar}+\frac{\iota p_{0}}{\hbar}\left(q-q_{0}\right)\right),
\end{aligned}
$$

To calculate the time-dependent energy, fluctuation and energy population spectrum, Kumar et al., used a time evolved reduced density, defined as follows,

$\hat{\rho}_{t}=\operatorname{Tr}_{B}\left[\exp \left(-\frac{\iota}{\hbar} \hat{H} t\right) \hat{\rho_{0}} \exp \left(\frac{\iota}{\hbar} \hat{H} t\right)\right]$.

The time-dependent energy $\langle E(t)\rangle$, energy fluctuation $\langle\Delta E(t)\rangle^{2}$ and energy population spectrum $S(\omega, t)$ were defined as follows,

$\langle E(t)\rangle=\operatorname{Tr}\left[\exp \left(\frac{\iota}{\hbar} \hat{H} t\right) \hat{H}_{S} \exp \left(-\frac{\iota}{\hbar} \hat{H} t\right)\right]$,

where, $\hat{H}_{S}$ represents the Hamiltonian of system.

$\langle\Delta E(t)\rangle^{2}=\left\langle E^{2}(t)\right\rangle-\langle E(t)\rangle^{2}$,

and

$S(\omega, t)=\operatorname{Tr}_{S}\left[\delta\left(\omega-\frac{\hat{H}_{S}}{\hbar}\right) \hat{\rho}_{t}\right]$.

The full expressions of average energy $(\langle E(t)\rangle)$, energy fluctuation $\left(\langle\Delta E(t)\rangle^{2}\right)$ and energy population spectrum $(S(\omega, t))$ can be found in the Ref. ${ }^{7}$

In the above model, the system was assumed to be harmonic. It is a good approximation, if the system under consideration is in its ground vibrational state. But, as we move to higher vibrational states, anharmonicity becomes very important. There are different ways to incorporate anharmonicity in the system. One very common way is to replace the harmonic oscillator with Morse oscillator. ${ }^{32-34}$ Another way is to treat anharmonicity perturbatively. ${ }^{35}$ One common problem with both of these approaches is, that we lose the simplicity of harmonic model. In harmonic approximation, most of the integrals encountered, are analytically doable, whereas, the same is not possible with Morse or perturbative treatment. In this context, the approach used by Bader et al., seems to be very useful. They replaced the anharmonic oscillator with an effective harmonic system, defined by an instantaneous frequency $(\omega(E))$, action $(J(E))$ and oscillator period $(\mathrm{T}(\mathrm{E}))$ as follows, ${ }^{36}$

$T(E)=\frac{2 \pi}{\omega(E)}=\frac{d J(E)}{d E}$

Bader et al., derived an expression of the relaxation of the $\mathrm{J}(\mathrm{E})$ for a harmonic oscillator linearly coupled to a harmonic bath, ${ }^{36}$

$\frac{d}{d t} \ln \left[J(E)-J_{e q}\right]=-\overline{\gamma^{\prime}}(\omega(E))$

Here, $\bar{\gamma}^{\prime}$ is real part of Fourier-Laplace transform of stochastic force. For a Morse oscillator, defined by the following potential,

$V(q)=D_{0}\left(1-e^{-\alpha q}\right)^{2}$

energy can be written as follows,

$E=\left(\omega_{0} /(2 \pi)\right) J(1-\chi J)$

Where, $\omega_{0}$ is harmonic frequency associated with Morse vibrations and $\chi$ is anharmonicity constant and can be defined in terms of dissociation energy $D_{0}$ as follows,

$D_{0}=\frac{\omega_{0}}{8 \pi \chi}$

Using Eqs. 9 and 12, the instantaneous frequency of anharmonic system can be obtained, ${ }^{36}$

$\omega(E)=\omega_{0}(1-2 \chi J(E))$

Consequently, in this model, incorporating the anharmonicity amounts to calculating the instantaneous frequency at each energy, which in turn depends upon how action relax with time. For exponential friction with decay time $\tau$, the following expression was found for relaxation of action, ${ }^{36}$

$\frac{d}{d t} \ln \left[J(t)-J_{e q}\right]=-\frac{\gamma}{\left[1+\tau^{2} \omega_{0}^{2}(1-2 \chi J)^{2}\right]}$

Where,

$J_{e q}=\frac{E_{e q}}{\omega_{0} \sqrt{1-\frac{E_{e q}}{D_{0}}}}$,

Here, $E_{e q}$ is the average energy of a harmonic oscillator at equilibrium.

This particular model is well-suited for our purpose. We just need to compute the harmonic frequency at each energy. The main advantage of this approach is that we can use equations derived for average energy, fluctuation and population spectrum by Kumar et al. ${ }^{7}$ as such, with an energy dependent harmonic frequency. It is worth mentioning that the analytic expression obtained by Kumar and Pollak was due to the exact correspondence between classical and quantum mechanics for harmonic oscillators. Interestingly, for anharmonic oscillator this correspondence fails. In the present method, we assumed that the relaxation of instantaneous harmonic frequency due to anharmonicity is slower compared to the relaxation due to system-bath coupling. Under these circumstances, we can treat the relaxation time of frequency due to anharmonicity 
as a parameter rather a variable and for each value of this parameter we can assume the system to be harmonic oscillator. Therefore, it is a kind of adiabatic approximation. In the next section, we have used this approach to shed light on effect of anharmonicity on energy relaxation of a dissipative harmonic system.

\section{Numerical application}

Similar to an earlier work, ${ }^{7}$ we have also used the following exponential memory function to define spectral density of bath.

$\gamma(t)=\frac{\gamma}{\tau} \exp (-t / \tau)$

Where, $\gamma$ is a friction strength parameter and $\tau$ is the memory time. To compute the frequency and fluctuation, we have used the experimentally measured frequencies of the $v_{C=C}$ mode of trans-stilbene i.e., $\Omega_{0}=1570 \mathrm{~cm}^{-1}$ (ground state) and $\Omega_{1}=1596 \mathrm{~cm}^{-1}$ (excited state). ${ }^{26,37}$ The numerical values of other parameters are chosen in such a way that, it can mimic the FSRS experiment.

\subsection{Frequency}

In the FSRS experiment, the initial value of frequency for the peak position of $v_{C=C}$ band in $S_{1}$ state, was found to be blue shifted as compared to the equilibrium value. The magnitude of this frequency shift depends strongly upon the excitation wavelength. For the lowest excitation wavelength $(267 \mathrm{~nm})$, the frequency shift was found to be maximum $\left(\sim 30 \mathrm{~cm}^{-1}\right)$. As the excitation wavelength increases, the shift decreases monotonically and for $318 \mathrm{~nm}$, almost no change in the frequency shift was observed in experiment. In the theoretical model employed in the present work, the peak of experimental band corresponds to the instantaneous harmonic frequency (defined in Eq. (14)). The time evolution of instantaneous harmonic frequency for $v_{C=C}$ mode of trans-stilbene is depicted in Figure 1. It is evident from the Figure 1 that, similar to the experimental observation, not only the frequency values increase with time but also the magnitude in shifts depend upon excitation wavelength. In accord with experimental observation, for $267 \mathrm{~nm}$ excitation, this change was found to be maximum $\left(\sim 30 \mathrm{~cm}^{-1}\right)$, while for $318 \mathrm{~nm}$ this change was negligible. It is also worth mentioning that, the experimental frequency values were reported at 0 ps and 100 ps. Therefore, we have compared the computed frequency values only at 0 ps and 100 ps. It is evident from Table 1, that the computed frequency shifts are in

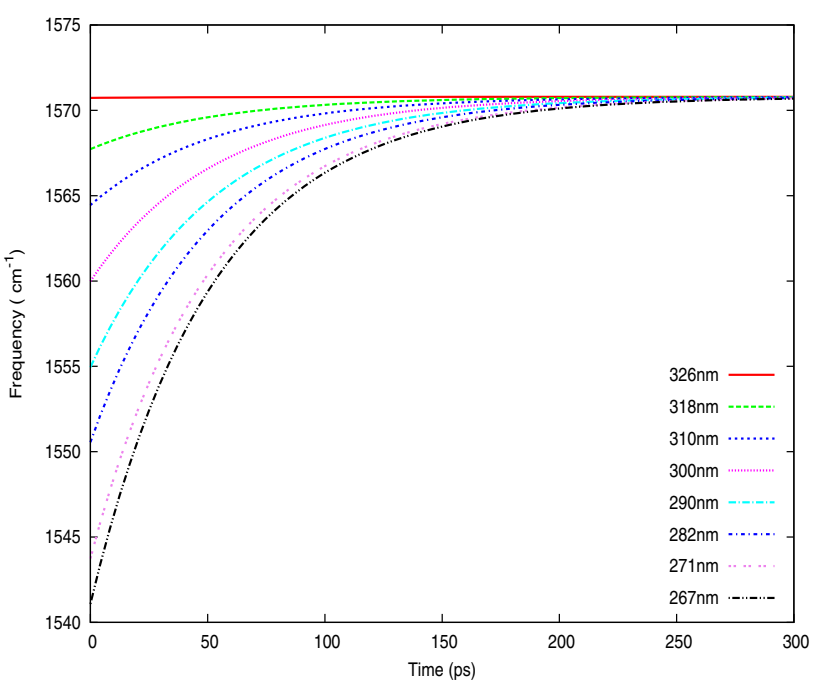

Figure 1. Time evolution of instantaneous harmonic frequency of $v_{C=C}$ band of trans-stilbene in $S_{1}$ state at different excitation wavelength ( $\tau$ and $\gamma$ are taken to be $10 \mathrm{fs}$ and 40.04 $\mathrm{cm}^{-1}$ respectively).

good agreement with experimental values reported by Kovalenko et al. ${ }^{26}$

\subsection{Variance}

As mentioned in introduction, another interesting observation in the FSRS experiment was, a non-linear time evolution of full width at half maximum (FWHM) for $v_{C=C}$ band of trans-stilbene in vibrationally excited $S_{1}$ state. Kumar et al., computed the $\langle\Delta E(t)\rangle$ for various vibrationally excited states at $294 \mathrm{~K}$. They found a linear exponential decay of $\langle\Delta E(t)\rangle$ for all the excited states. We believe that the non-linear decay of FWHM found for higher vibrational excited states, not only depend upon initial, but also on equilibrium values of FWHM. It is important to note that there is a difference between energy fluctuation calculated by Eq. (7) and the experimentally measured FWHM. The $\langle\Delta E(t)\rangle$ corresponds to the evolution in time of the whole vibrational population of $v_{C=C}$ mode, which contributes to the initial state of system i.e., Gaussian wavefunction, defined in Eq. (4). Whereas, experimentally measured FWHM corresponds to evolution of instantaneous fluctuations in single $v_{C=C}$ mode of trans-stilbene. Consequently, computed values of initial energy fluctuation $(\langle\Delta E(t)\rangle)$ are found to be much higher as compared to FWHM measured by FSRS experiment. For example, at $267 \mathrm{~nm}$ excitation wavelength, the value of $\langle\Delta E(t)\rangle$ at $t=0$ ps was computed to be $3285 \mathrm{~cm}^{-1}$, whereas, the experimentally measured value of FWHM was $56 \mathrm{~cm}^{-1}$. On the other hand, equilibrium value of FWHM as well as theoretically computed value of $\langle\Delta E(t)\rangle$, both depend 
Table 1. Comparison of theoretically calculated instantaneous harmonic frequencies of $v_{C=C}$ band of trans-stilbene in $S_{1}$ state with experimental values at initial and equilibrium time at different initial energies ( $\tau$ and $\gamma$ are taken to be $10 \mathrm{fs}$ and $40.04 \mathrm{~cm}^{-1}$ respectively).

\begin{tabular}{|c|c|c|c|c|}
\hline \multirow[t]{2}{*}{ Excitation wavelength (nm) } & \multicolumn{2}{|c|}{ Theoretical $\left(\mathrm{cm}^{-1}\right)$} & \multicolumn{2}{|c|}{ Experimental $\left(\mathrm{cm}^{-1}\right)$} \\
\hline & Initial & Equilibrium & Initial & Equilibrium \\
\hline 267 & 1541 & 1570.5 & 1541 & 1572.1 \\
\hline 271 & 1544 & 1570.5 & 1545 & 1569.2 \\
\hline 282 & 1550 & 1570.5 & 1548 & 1569.2 \\
\hline 290 & 1554 & 1570.5 & 1551 & 1568.2 \\
\hline 300 & 1560 & 1570.5 & 1559 & 1568.2 \\
\hline 310 & 1564 & 1570.5 & 1564 & 1570.1 \\
\hline 318 & 1568 & 1570.5 & 1568 & 1568.2 \\
\hline 326 & 1570.5 & 1570.5 & 1570.2 & 1570.2 \\
\hline
\end{tabular}

on temperature. Consequently, they are found to be of the same order. Therefore, the shape of experimental evolution of FWHM can only be obtained, if similar to FSRS experiment, computed equilibrium values of $\langle\Delta E(t)\rangle$, are of same order as initial values. As asymptotic values of $\langle\Delta E(t)\rangle$ depend only on temperature, we computed variances at those temperatures, where the value of $\langle\Delta E(t)\rangle$ at $t=\infty$ become comparable to $\langle\Delta E(t)\rangle$ at $t=0 \mathrm{ps}$.

For example at $3900 \mathrm{~K},\langle\Delta E(t)\rangle_{t=\infty}$ is $\sim 2678 \mathrm{~cm}^{-1}$ which is of same order as $\langle\Delta E(t)\rangle$ at $0 \mathrm{ps}$. Consequently, now the $\langle\Delta E(t)\rangle$ relaxed non-linearly (Figure 2). It is also worth mentioning that the $3900 \mathrm{~K}$ did not correspond to the bath temperature of experiment. To compare the $\langle\Delta E(t)\rangle$ of present work with FWHS of experiment, we need a proper scaling. It is shown later that after proper scaling, it corresponds to $\sim 280 \mathrm{~K}$ of bath temperature. Figure 2 also contains, $\langle\Delta E(t)\rangle$ curve after including the anharmonicity effect. Fitting of frequency data suggested the anharmonicity constant to be around 0.0119. It is evident from Figure 2(b) and (d) that, although anharmonicity makes the relaxation faster but the change is very small. A higher value of anharmonicity 0.0439 has also been used to see any effect of anharmonicity on energy relaxation. It is clearly evident that even with these values, an absolute change in $\langle\Delta E(t)\rangle$ curve is very small $\left(1.54 \mathrm{~cm}^{-1}\right)$. Therefore, we believe that anharmonicity does not affect the relaxation process directly. At this point, it is also worth investigating that, within the limitation of present theoretical model, why does the $\langle\Delta E(t)\rangle$ relax non-linearly at high temperature?

To understand it, we have plotted the energy population spectrum (defined in Eq. (8)) for $3900 \mathrm{~K}$ at various time. It is evident from Figure 3 that initial population of various vibrational states are not in Boltzmann distribution. This non-equilibrium distribution finally evolves to an equilibrium Boltzmann population distribution. Consequently, at $\mathrm{t}=0 \mathrm{ps}$, higher vibrationally excited states are more populated, whereas, at the equilibrium there is an exponential decay of population as we move from ground state to higher excited state. It is quite possible that, at some intermediate time, the higher excited state population did not vanish completely, and at the same time, a few new population at lower energy state started to build up. Consequently, $\langle\Delta E(t)\rangle$, which is a measure of total width of populated states, increases at intermediate time.

Now as far as FSRS experiment is concerned, a direct comparison between FSRS experiment and our theoretical model is not possible, as theoretical values of $\langle\Delta E(t)\rangle$ are much higher as compared to experimental FWHM. To make the $\langle\Delta E(t)\rangle$ comparable to experimental FWHM, we have scaled the theoretically calculated values of energy fluctuation. The basic idea behind scaling is to find appropriate temperature for various excited wavelength in such a way that, it resembles the experimental FWHM. Then, we divide the whole variance data by that number which makes the initial value of $\langle\Delta E(t)\rangle$ same to the corresponding FWHM value found in FSRS experiment. Figure 4 depicts the case, where we have kept the ratio of initial and final $\langle\Delta E(t)\rangle$ values identical to the corresponding values of FWHM. As expected, the initial and final values do match with experiment. But the initial rise in the scaled $\langle\Delta E(t)\rangle$ values are different from experimental values. For $267 \mathrm{~nm}$ wavelength, the FWHM, starts from $\sim 56$ $\mathrm{cm}^{-1}$ and increases upto $\sim 65 \mathrm{~cm}^{-1}$, whereas, theoretically scaled $\langle\Delta E(t)\rangle$ increases upto $\sim 60 \mathrm{~cm}^{-1}$. This discrepancy between experimental and theoretical values decreases as the excitation wavelength increases. For $282 \mathrm{~nm}$ and $290 \mathrm{~nm}$, the theoretical and experimental curves are in good agreement. For $300 \mathrm{~nm}$ wavelength, the difference between experimental and 


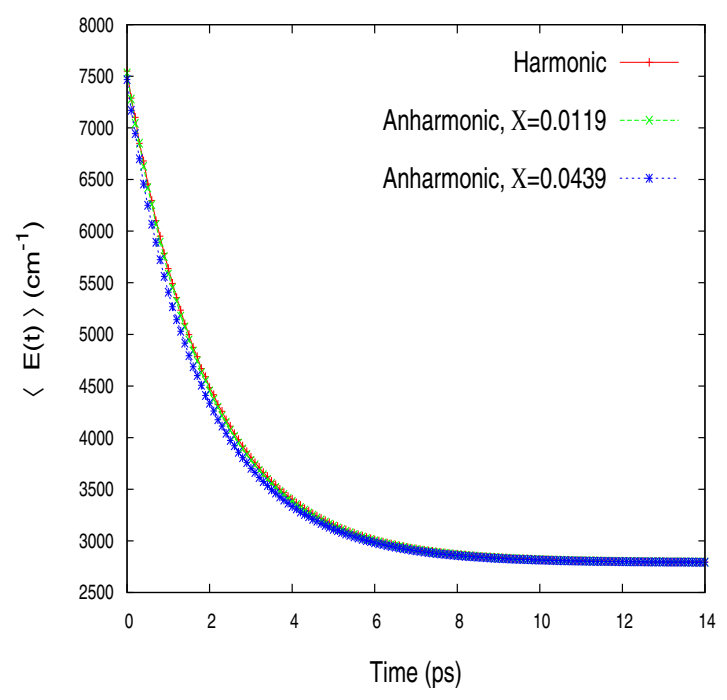

(a)

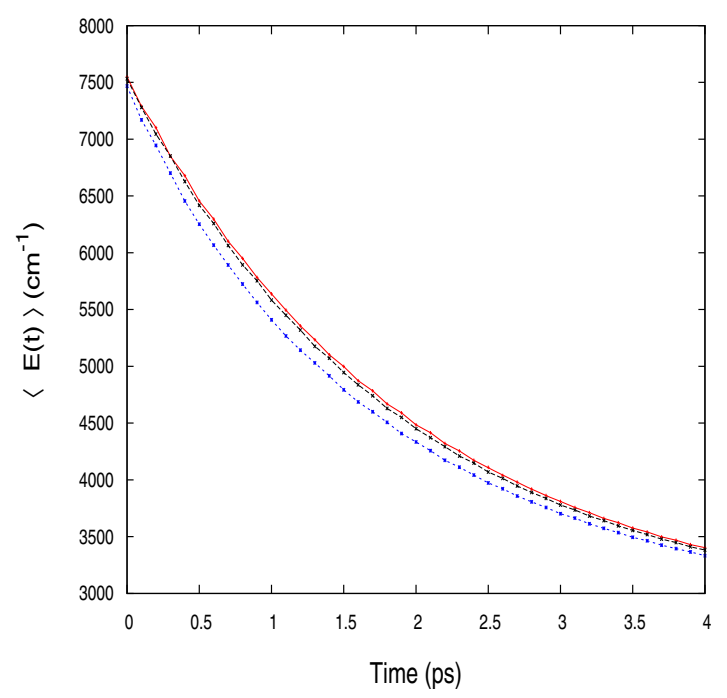

(c)

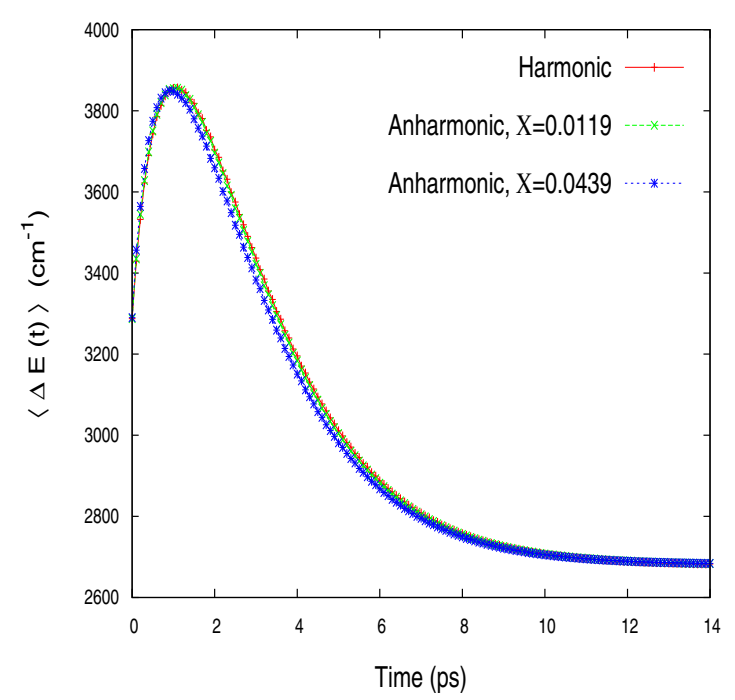

(b)

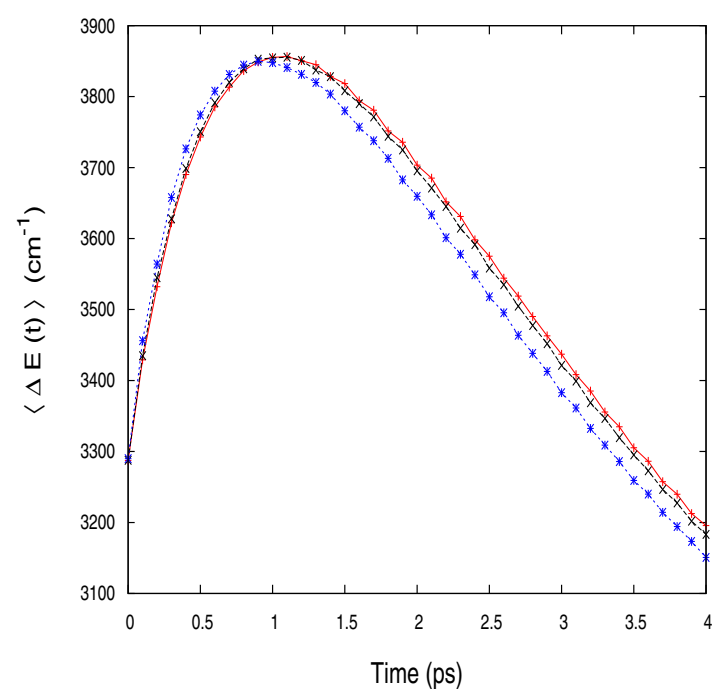

(d)

Figure 2. Effect of anharmonicity on average energy relaxation (panel a) and variance (panel b) at a constant initial energy $\left(p_{0}\right)$ of 7.84. Panel (c) and (d) are the enlarged portion of figure (a) and (b) respectively. The bath temperature (T) is $3900 \mathrm{~K}$, memory time $(\tau)$ is $10 \mathrm{fs}$ and, coupling constant $(\gamma)$ is $26.8 \mathrm{~cm}^{-1}$.

theoretical curves becomes significant again. There are two major sources of this discrepancy:-

- Besides, the interaction with solvent, IVR is another factor which contribute to the shape of relaxation curves.

- The values of $\gamma$ and $\tau$ need not be same for all the states.

As far as IVR is concerned, it will depend upon how strongly the $v_{C=C}$ couple with other modes, which in turn depends upon anharmonicity of $v_{C=C}$ mode. Consequently, it becomes more important for higher vibrational states and as we move towards ground vibrational state, the anharmonic effect reduces. It might be the reason behind higher discrepancy between experimental and theoretical curve, for lower excitation wavelength as compared to the case when excitation wavelength was high. The timescale of IVR is also expected to be faster than solvent relaxation timescale. Consequently, it is supposed to dominate at an early stage of relaxation. Unfortunately, IVR is not incorporated in the present theoretical model. If we assume that IVR acts as a heat bath, then it is quite possible that by varying the temperature, we can mimic the IVR dynamics within the limitation of our model. Therefore, if we wish to get closer to experimental curve in a short timescale, we need to vary the temperature. 


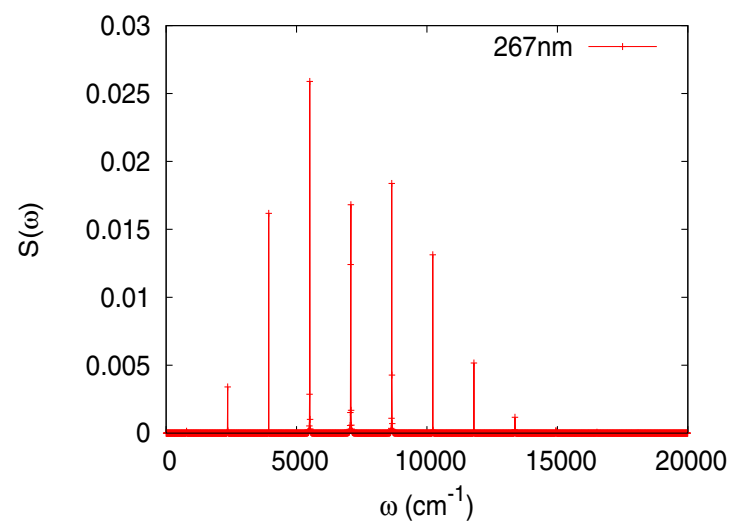

(a) $0 \mathrm{ps}$

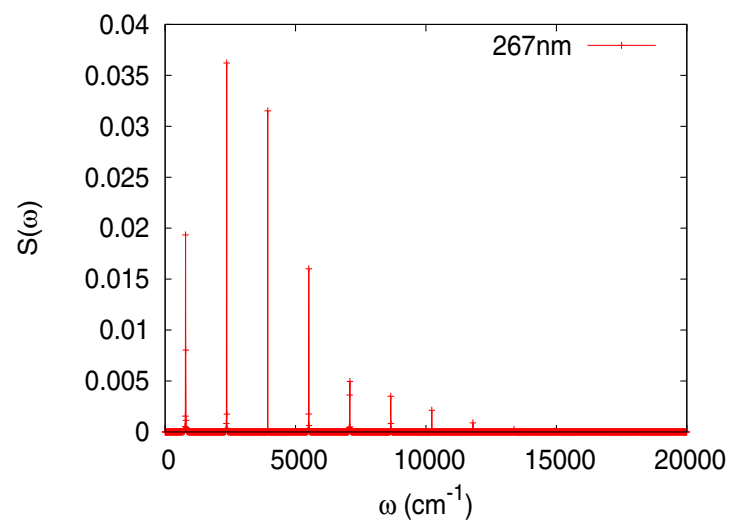

(c) $1 \mathrm{ps}$

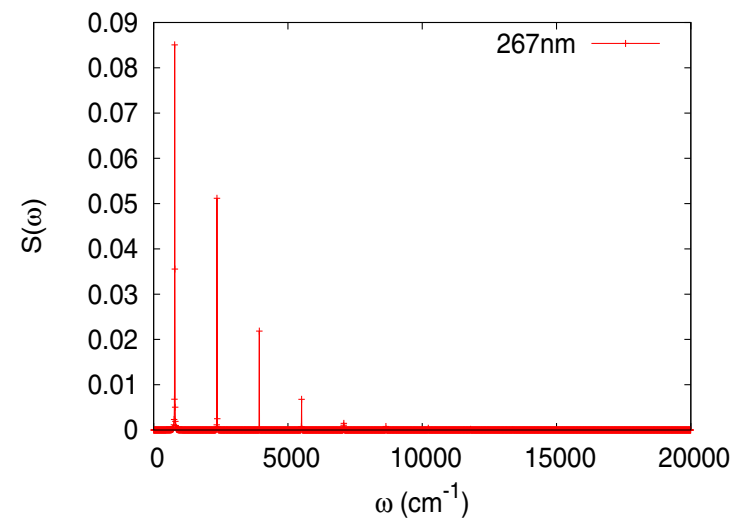

(e) $3 \mathrm{ps}$

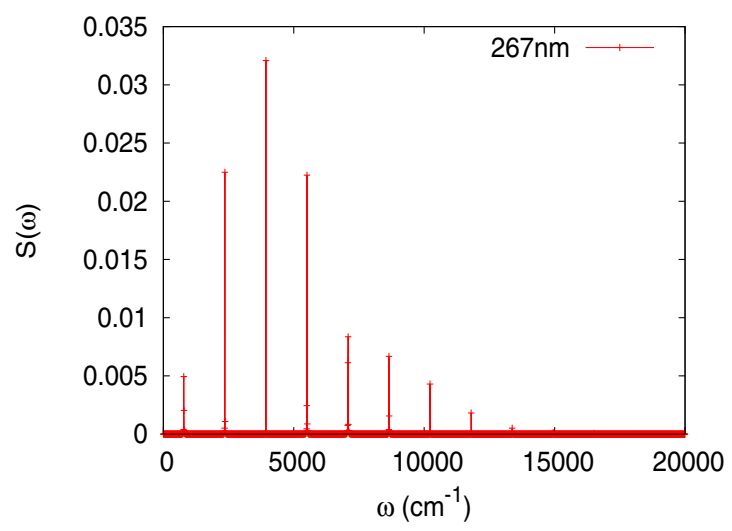

(b) $0.5 \mathrm{ps}$

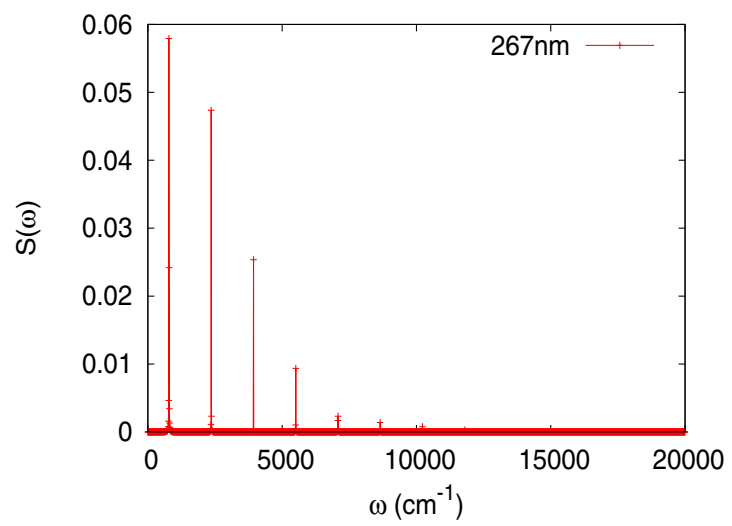

(d) $2 \mathrm{ps}$

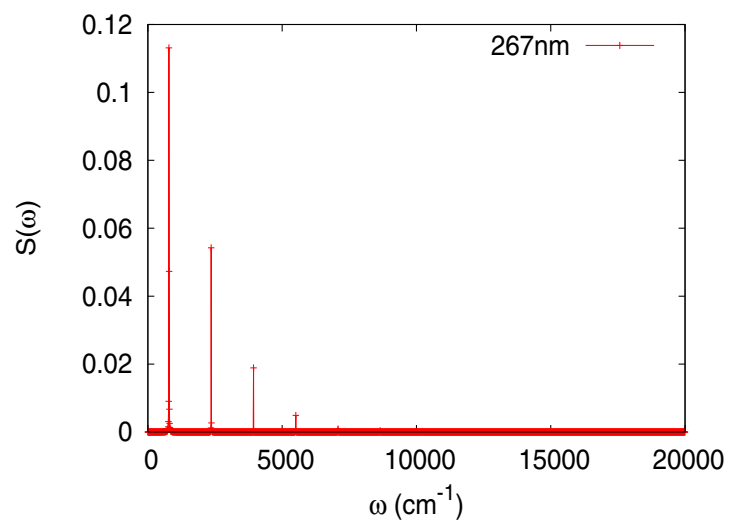

(f) $15 \mathrm{ps}$

Figure 3. The time dependent vibrational population of the dissipative harmonic oscillator at a bath temperature of $3900 \mathrm{~K}$, other parameters are same as in Figure 2.

The key reason behind the second source of discrepancy is also anharmonicity. As we moved to higher vibrational states, anharmonicity increases and as expected, strength of solute and solvent interaction also change. The change in $\tau$ with changing the excitation energy is also related to anharmonicity. The value of $\tau$ is inversely proportional to corresponding frequency of vibration. As the anharmonicity increases, the effective frequency decreases and consequently $\tau$ increases.
Keeping all the factors discussed above, Figure 5 depicts the best fitted theoretical curves. All the information about scaling is given in Table 2. In these curves, our focus was initial shape of FWHM curves. Therefore, we have varied the values of temperature, $\gamma$ and $\tau$, in such a way that, it can mimic the experimental curves as close as possible in the initial time ( 0 ps -10 ps) evolution. The following points are worth mentioning regarding Figure 5. 


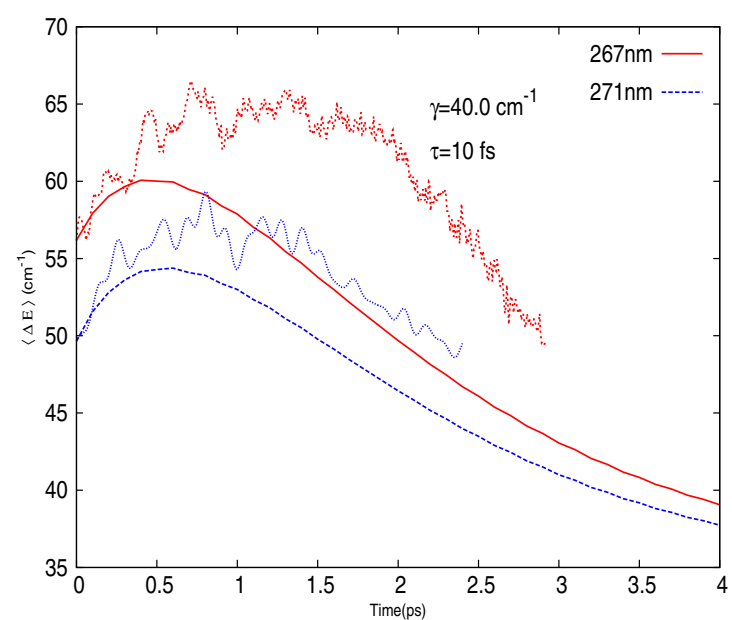

(a)

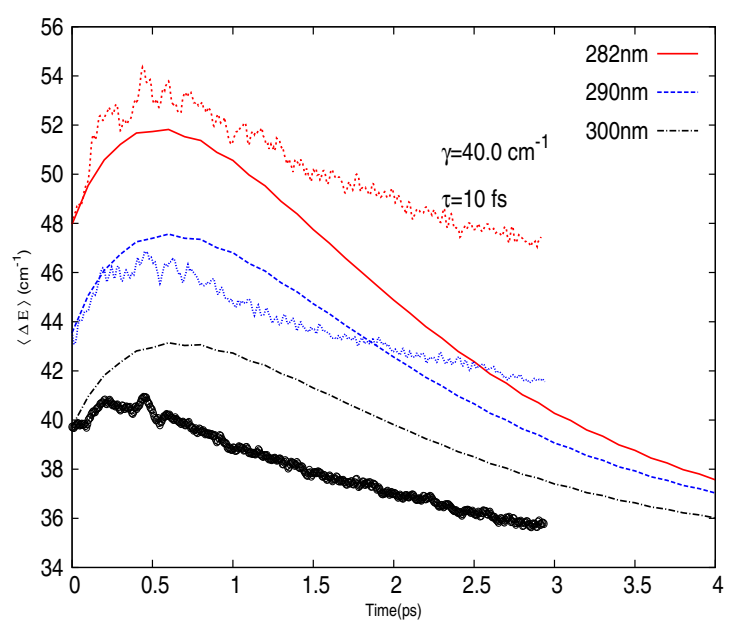

(b)

Figure 4. Comparison of experimental (dotted line) and theoretical (solid line) relaxation of the scaled variance $(\langle\Delta E\rangle)$ of $v_{C=C}$ band of trans-stilbene in $S_{1}$ state at $267 \mathrm{~nm}$ and $271 \mathrm{~nm}$ excitation wavelength (panel a) as well as at $282 \mathrm{~nm}, 290 \mathrm{~nm}$ and $300 \mathrm{~nm}$ (panel b).

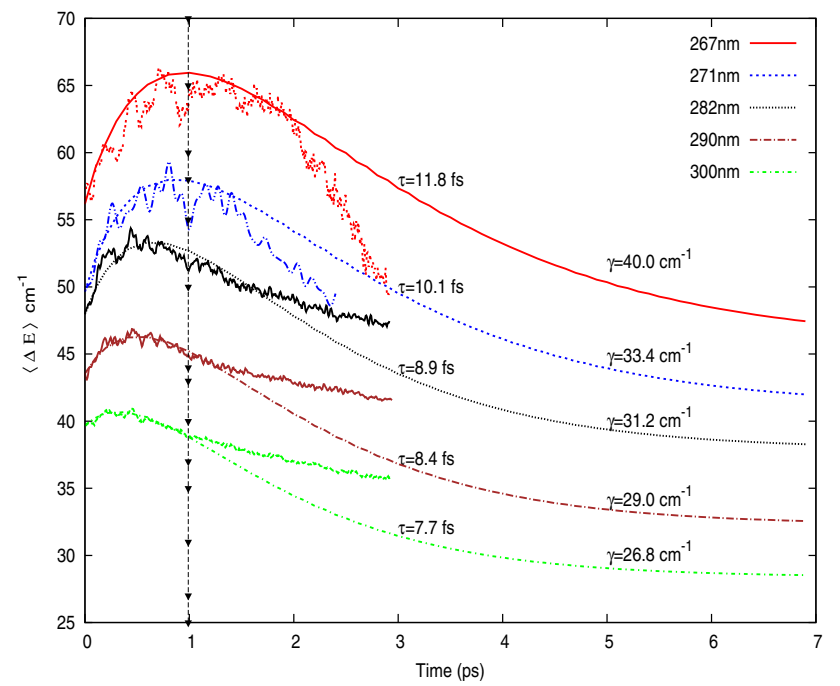

Figure 5. Comparison of theoretically calculated variance of trans-stilbene (solid lines) with FSRS experiment results (dotted lines) with different excitation energy.

- The asymptotic values of scaled $\langle\Delta E(t)\rangle_{t=\infty}$ decreases as the excitation wavelength increases (see Table 3), For $267 \mathrm{~nm}$, the corresponding scaled $\langle\Delta E(t)\rangle_{t=\infty}$ were computed to be $\sim 46 \mathrm{~cm}^{-1}$ which become as less as $\sim 28 \mathrm{~cm}^{-1}$ for $300 \mathrm{~nm}$ excitation wavelength.

- The values of $\gamma$ also decreases with increasing wavelength. For $267 \mathrm{~nm}$ it is $\sim 40 \mathrm{~cm}^{-1}$ whereas, for 300 $\mathrm{nm}$ it is $\sim 27 \mathrm{~cm}^{-1}$.

- The values of $\tau$ also decreases with increasing wavelength. For $267 \mathrm{~nm} \tau$ is $\sim 75 \mathrm{fs}$ whereas, for $300 \mathrm{~nm}$ it is $\sim 49 \mathrm{fs}$.
A decrease in fitted $\langle\Delta E(t)\rangle_{t=\infty}$ as we increase the wavelength indicated that, if we replace IVR dynamics with an effective bath dynamics, then in higher vibrational state, temperature of this bath is relatively higher than lower vibrational state. It is worth mentioning that only in a statistical limit for IVR one should associate the temperature with it. Stilbene is a benchmark example for non-statistical IVR. Therefore varying temperature here is just a mathematical trick. It is interesting to note that, IVR is present in gas as well as in solution phase. The present analysis suggests that for lower vibrational states, IVR may cool the excited molecules (as compared to solution temperature), which may get reheated by solvent molecules. Therefore, our present analysis is in accord with Pollak and co-workers hypothesis. ${ }^{18,19,22}$ Interestingly, the present analysis also suggests that at a higher vibrational excited state, excited molecule can get heated by IVR bath, which in longer timescale may get cooled by solvent. One more important observation in FSRS experiment was the time of maximum; in time evolution of FWHM, it was different for different vibrational excitation energy. For higher excited states it was more, and then it decreases monotonically as the excitation wavelength increases. It can be inferred from Figure 5 that our fitted parameters reproduce this trend successfully. We believe that a shift in time of maximum was due to the change in the value of $\tau$ for different state. As the excitation wavelength increase, value of $\tau$ decrease. As earlier explained, this change in $\tau$ is related to the anharmonicity. Therefore we can attribute this experimental observation also to the anharmonicity. 
Table 2. Values of memory time $(\tau)$ and coupling constant $(\gamma)$ corresponding to different temperatures and excitation wavelengths used to fit the theoretical date in figure 5 .

\begin{tabular}{lcrrc}
\hline Temperature $(\mathrm{K})$ & Excitation wavelength $(\mathrm{nm})$ & $\tau(\mathrm{fs})$ & $\gamma\left(\mathrm{cm}^{-1}\right)$ & Scaling factor \\
\hline 3900 & 267 & 11.83 & 40.04 & 58.5 \\
3800 & 271 & 10.14 & 33.44 & 63.5 \\
3200 & 282 & 8.93 & 31.24 & 57.6 \\
2700 & 290 & 8.45 & 29.04 & 56.6 \\
2200 & 300 & 7.72 & 26.84 & 51.7 \\
\hline
\end{tabular}

Table 3. Asymptotic values of scaled variance corresponding to different excitation wavelength.

\begin{tabular}{lc}
\hline Excitation wavelength $(\mathrm{nm})$ & Asymptotic value $\left(\mathrm{cm}^{-1}\right)$ \\
\hline 267 & 45.81 \\
271 & 40.99 \\
282 & 37.92 \\
290 & 32.30 \\
300 & 27.90 \\
\hline
\end{tabular}

\section{Conclusions}

Quantum dissipative harmonic oscillator has previously been used as a model to shed light on optical cooling of trans-stilbene molecule. We have incorporated the anharmonicity in this model and shown that, anharmonicity plays a crucial role in energy relaxation of vibrationally excited states. It has been conjectured that anharmonicity affects the relaxation process indirectly, (By modifying coupling mode and memory time scale) rather directly (By modifying effective harmonic frequency). The present analysis also suggests that at an initial stage, IVR plays a key role in changing the effective temperature of optically excited molecule. If we treat IVR also as heat bath, then for higher vibrationally excited state, the temperature of IVR bath is more as compared to the lower vibrational state. It is suggested that similar to the solution phase, in the gas phase also, the excited molecule may get reheated by IVR.

Although, the present model tried to explain several experimental observations, it still has a few shortcomings. It does not include IVR phenomenon directly, which might play an important role at vibrationally higher excited state. The present model also does not contain the width of an individual spectral line as there is no stochastic component in the frequency, in the Hamiltonian employed for the present work.

Another limitation of the present analysis is the assumption of linear coupling between system and bath. It is known that in more realistic model this coupling can be nonlinear. It will be interesting to see which is going to be more important- the correction due to the anharmonicity or the correction due to nonlinear system-bath coupling. Our work in the future will focus on these issues.

\section{Acknowledgements}

RK acknowledges DST, Govt. of India for financial assistance. PK acknowledges DST, Govt. of India for the financial support through sanctioned project [No. ECR/2016/000279]. We also thank Prof N. Ernsting for sending us experimental data of Figure 5.

\section{References}

1. Pigliucci A, Duvanel G, Daku L M L and Vauthey E 2007 Investigation of the Influence of Solute-Solvent Interactions on the Vibrational Energy Relaxation Dynamics of Large Molecules in Liquids J. Phys. Chem. A 1116135

2. Heitz R, Veit M, Ledentsov N N, Hoffmann A, Bimberg D, Ustinov V M, Kopev P S and Alferov Z I 1997 Energy relaxation by multiphonon processes in InAs/GaAs quantum dots Phys. Rev. B 5610435

3. Morisaki H, Koretsune T, Hotta C, Takeya J, Kimura T and Wakabayashi Y 2014 Large surface relaxation in the organic semiconductor tetracene Nat. Commun. 55400

4. Ishizaki A and Fleming G R 2012 Quantum coherence in photosynthetic light harvesting Annu. Rev. Condens. Matter Phys. 3333

5. von Lintig J, Kiser P D, Golczak M and Palczewski K 2010 The biochemical and structural basis for trans-tocis isomerization of retinoids in the chemistry of vision Trends Biochem. Sci. 35400

6. Kukura P, McCamant D W, Yoon S, Wandschneider D B and Mathies R A 2005 Structural observation of the primary isomerization in vision with femtosecondstimulated Raman Science $\mathbf{3 1 0} 1006$

7. Kumar P and Pollak E 2014 Energy relaxation of a dissipative quantum oscillator J. Chem. Phys. 141234509

8. Pollak E, Shao J and Zhang D H 2008 Effects of initial correlations on the dynamics of dissipative systems Phys. Rev. E 77021107

9. Levine A M, Shapiro M and Pollak E 1988 Hamiltonian theory for vibrational dephasing rates of small molecules in liquids J. Chem. Phys. 881959 
10. Rips I and Pollak E 1990 Quantum Kramers model: Solution of the turnover problem Phys. Rev. A 415366

11. Waldeck D H 1991 Photoisomerization dynamics of stilbenes Chem. Rev. 91415

12. Hochstrasser R M 1980 Picosecond processes in the isomerism of stilbenes Pure Appl. Chem. 522683

13. Saltiel J and Charlton J 1980 Organic Chemistry: A Series of Monographs Vol. 42 (New York: Elsevier) p. 25

14. Meyer A, Schroeder J and Troe J 1999 Photoisomerization of trans-stilbene in moderately compressed gases: Pressure-dependent effective barriers J. Phys. Chem. A 10310528

15. Sundström V and Gillbro T 1984 Dynamics of the isomerization of trans-stilbene in n-alcohols studied by ultraviolet picosecond absorption recovery Chem. Phys. Lett. 109538

16. Courtney S and Fleming G 1985 Photoisomerization of stilbene in low viscosity solvents: comparison of isolated and solvated molecules J. Chem. Phys. 83215

17. Balk M W and Fleming G R 1986 Unimolecular reactions in isolated and collisional systems: Is the transitionstate rate an upper limit for the isomerization of stilbene? J. Phys. Chem. 903975

18. Gershinsky G and Pollak E 1997 Unimolecular reactions in the gas and liquid phases: A possible resolution to the puzzles of the trans-stilbene isomerization J. Chem. Phys. 107812

19. Pollak E, Talkner P and Berezhkovskii A 1997 A theory for nonisothermal unimolecular reaction rates J. Chem. Phys. 1073542

20. Courtney S H, Balk M W, Philips L A, Webb S P, Yang D, Levy D H and Fleming G R 1988 Unimolecular reactions in isolated and collisional systems: deuterium isotope effect in the photoisomerization of stilbene J. Chem. Phys. 896697

21. Troe J 1985 Quantitative analysis of photoisomerization rates in trans-stilbene and 4-methyl-trans-stilbene Chem. Phys. Lett. 114241

22. Nordholm S 1989 Photoisomerization of stilbene-a theoretical study of deuteration shifts and limited internal vibrational redistribution J. Chem. Phys. 137109

23. Wadi H and Pollak E 1999 Theory of laser cooling of polyatomic molecules in an electronically excited state J. Chem. Phys. 11011890

24. Tatchen J and Pollak E 2008 Ab initio spectroscopy and photoinduced cooling of the trans-stilbene molecule $J$. Chem. Phys. 128164303
25. Hamaguchi H-O 1996 Solvent-induced dynamic polarization and vibrational dephasing of electronically excited molecules Mol. Phys. 89463

26. Kovalenko S, Dobryakov A, Pollak E and Ernsting N 2013 Communication: Optical cooling of trans-stilbene J. Chem. Phys. 139011101

27. Iwata K and Hamaguchi H-o 1992 Picosecond structural relaxation of $\mathrm{S} 1$ trans-stilbene in solution as revealed by time-resolved Raman spectroscopy Chem. Phys. Lett. 196462

28. Iwata K, Ozawa R and Hamaguchi H-o 2002 Analysis of the Solvent-and Temperature-Dependent Raman Spectral Changes of S1 trans-Stilbene and the Mechanism of the trans to cis Isomerization: Dynamic Polarization Model of Vibrational Dephasing and the CC Double-Bond Rotation J. Phys. Chem. A 106 3614

29. Oxtoby D W 1979 Dephasing of molecular vibrations in liquids $A d v$. Chem. Phys. $\mathbf{4 0} 1$

30. Leitner D M, Levine B, Quenneville J, Martínez T J and Wolynes P G 2003 Quantum energy flow and trans-stilbene photoisomerization: an example of a non-RRKM reaction J. Chem. Phys. A $\mathbf{1 0 7}$ 10706

31. Weston R E and Barker J R 2006 On modeling the pressure-dependent photoisomerization of trans-stilbene by including slow intramolecular vibrational energy redistribution J. Chem. Phys. A 1107888

32. Tuckerman M and Berne B 1993 Vibrational relaxation in simple fluids: Comparison of theory and simulation $J$. Chem. Phys. 987301

33. Dove J and Jones D G 1971 Numerical Calculation of Vibrational Relaxation and Dissociation for a Quantum Anharmonic Oscillator J. Chem. Phys. 551531

34. Ishizaki A and Tanimura Y 2006 Modeling vibrational dephasing and energy relaxation of intramolecular anharmonic modes for multidimensional infrared spectroscopies J. Chem. Phys. 125084501

35. Bader J S and Berne B 1994 Quantum and classical relaxation rates from classical simulations J. Chem. Phys. 100 8359

36. Bader J S, Berne B, Pollak E and Hänggi P 1996 The energy relaxation of a nonlinear oscillator coupled to a linear bath J. Chem. Phys. 1041111

37. Dobryakov A, Ioffe I, Granovsky A, Ernsting $\mathrm{N}$ and Kovalenko S 2012 Femtosecond Raman spectra of cisstilbene and trans-stilbene with isotopomers in solution J. Chem. Phys. 137244505 